\title{
A Low-Complexity Subcarrier-Power Allocation Scheme for Frequency-Division Multiple-Access Systems
}

\author{
Tingting Liu, Student Member, IEEE, Chenyang Yang, Senior Member, IEEE, \\ and Lie-Liang Yang, Senior Member, IEEE
}

\begin{abstract}
This letter aims to design a low-complexity subcarrier-power allocation scheme to improve the communication reliability of various types of frequency-division multipleaccess (FDMA) systems. Both uplink and downlink are considered. Specifically, a low-complexity worst subcarrier avoiding (WSA) subcarrier-allocation scheme is proposed, in order to avoid assigning users the subcarriers experiencing severe fading. After the subcarrier-allocation, channel-inversion assisted powerallocation is employed to assign the subcarriers the corresponding power. Our studies and simulation results show that the achievable error performance of the FDMA systems employing the proposed subcarrier-power allocation algorithm is independent of the multiplexing method. The proposed algorithm outperforms the existing subcarrier-power allocation algorithms that have a similar complexity as the proposed one.
\end{abstract}

Index Terms-Subcarrier-allocation, power-allocation, FDMA, SC-FDMA, OFDMA, uplink, downlink.

\section{INTRODUCTION}

$\mathbf{T}$ HE ever-growing wireless multimedia services demand reliable high-data rate communications. The FDMA techniques, which include single-carrier FDMA (SC-FDMA), orthogonal FDMA (OFDMA), etc., have been recognized as the most promising candidates for delivering high-data rate multimedia services in the future generations of wireless communications systems. This is because the FDMA techniques are capable of providing high-flexibility multiple-access. With the aid of the dynamic resource allocation, which may include subcarrier-, power-, or/and rate-allocation $[1,2]$, the FDMA techniques are also capable of achieving high spectralefficiency and providing high quality-of-services (QoS).

Given the total number of subcarriers of a FDMA system, the subcarrier allocation problem belongs to the family of classic resource assignment problems whose optimum solutions can be obtained by the famous Hungarian algorithm designed based on graph theory [3]. However, the optimum Hungarian algorithm has a high computational complexity, especially, when time-varying wireless channels are considered. Hence, it is highly important to design the low-complexity allocation algorithms that can offer competitive error performance to the optimum algorithm but with significantly lower implementational complexity. For this sake, in [4], a Greedy algorithm

Manuscript received June 19, 2009; revised December 11, 2009; accepted February 15, 2010. The associate editor coordinating the review of this letter and approving it for publication was M. Ardakani.

T. Liu and C. Yang are with the School of Electronics and Information Engineering, Beihang University, Beijing 100191, China (e-mail: ttliu@ee.buaa.edu.cn, cyyang@buaa.edu.cn). T. Liu was a visiting Ph.D. student at the School of Electronics and Computer Science, University of Southampton, SO17 1BJ, UK, from January 2009 to January 2010.

L.-L. Yang is with the School of Electronics and Computer Science, University of Southampton, SO17 1BJ, UK (e-mail: 1ly@ecs.soton.ac.uk).

Digital Object Identifier 10.1109/TWC.2010.05.090926 has been proposed, in which users select one-by-one their desired subcarriers from the available options based on the subcarriers' channel qualities. The Greedy algorithm has low complexity, however, users at the later stages are left less optional subcarriers to choose and may have to select some subcarriers with low channel qualities, which hence results in high error rate. The worst user first (WUF) Greedy algorithm proposed in [5] allows the users with worse average channel qualities to select their required subcarriers first. However, the performance improvement of the WUF Greedy algorithm over the Greedy algorithm is very limited. In [6] a so-called Maximal Greedy algorithm has been developed. In the context of this algorithm, first, several subcarrier allocations based on the Greedy algorithm are operated by ordering the users in different ways. Then, the allocation resulting in the best error performance is chosen as the final subcarrier-allocation. The study in [6] shows that the Maximal Greedy algorithm is capable of improving the error performance significantly, but at the cost of a significantly increase of complexity. Additionally, in $[7,8]$ the subcarrier-allocation problem has also been investigated, which uses the Greedy algorithm to find an initial allocation and, then, improves it by iteratively swapping a pair of subcarriers of two arbitrary users. This subcarrier-allocation method may achieve the near-optimal error performance after a sufficient number of iterations. However, its complexity may be extreme when the number of subcarriers and/or the number of iterations are high.

In this contribution, we propose a low-complexity subcarrier-power allocation scheme, which is suitable for the FDMA systems using various multiplexing approaches, such as SC-FDMA and OFDMA. The proposed subcarrierallocation scheme is worst subcarrier avoiding (WSA), while the power-allocation is based on channel-inversion. As our studies show, the WSA algorithm is capable of avoiding assigning users most of the subcarriers with low channel qualities. Hence, as our simulation results show, the error performance of the FDMA systems can be significantly improved in comparison with that of the FDMA systems employing the existing algorithms $[4,5]$, which have a similar complexity as the proposed WSA algorithm.

The remainder of the letter is organized as follows. Section II states the FDMA system model. Section III details the subcarrier-power allocation algorithm. Complexity is analyzed in Section IV. In Section V we provide some simulation results. Finally, in Section VI we state the conclusions.

\section{FDMA SYSTEM MODEL}

The FDMA system considered in this contribution employs $M$ number of orthogonal subcarriers to support $Q$ users, 
whose indexes form a set $\mathcal{Q}=\{0,1, \ldots, Q-1\}$. The $q$ th, $q \in \mathcal{Q}$, user is assigned $K_{q}$ subcarriers and $\sum_{q \in \mathcal{Q}} K_{q} \leq M$. The base station is assumed to employ ideal channel state information about the $M$ subcarrier channels associated with any of the $Q$ users. It is capable of allocating the corresponding subcarriers and power to the $Q$ users through reliable lowrate signalling channels, whenever necessary. For clarity, the variables and notations used in this letter are summarized as follows:

$M \quad$ number of subcarriers;

$\mathcal{M}$ set of subcarrier indexes defined as $\mathcal{M}=$ $\{0,1, \ldots, M-1\}$;

$Q \quad$ number of uplink or downlink users;

$K_{q} \quad$ number of data streams of user $q$;

$\mathcal{K}_{q} \quad$ set of indexes for the data streams of user $q$, which is defined as $\mathcal{K}_{q}=\left\{0,1, \ldots, K_{q}-1\right\}$;

$H_{q, j} \quad$ channel gain of subcarrier $j$ of user $q$;

$N_{j} \quad$ uplink noise power of subcarrier $j$;

$N_{q, j} \quad$ downlink noise power of subcarrier $j$ of user $q$;

$G_{q, j}$ channel quality of subcarrier $j$ of user $q$, which is defined as $G_{q, j}=\left|H_{q, j}\right|^{2} / N_{q, j}$ for downlink, while as $G_{q, j}=\left|H_{q, j}\right|^{2} / N_{j}$ for uplink;

$P_{q} \quad$ maximum uplink power allowed for user $q$;

$P \quad$ maximum downlink power allowed for all $Q$ users;

$P_{q, j} \quad$ transmission power on subcarrier $j$ of user $q$, which satisfies the constraint of $\sum_{j \in \mathcal{F}_{q}} P_{q, j} \leq P_{q}$ for uplink or $\sum_{q \in \mathcal{Q}} \sum_{j \in \mathcal{F}_{q}} P_{q, j} \leq P$ for downlink;

$\mathcal{F}_{q}$ a set containing the indexes of the subcarriers assigned to user $q$, which has the properties $\bigcup_{q \in \mathcal{Q}} \mathcal{F}_{q} \subseteq \mathcal{M}$ and, for any $q, j \in \mathcal{Q}$ and $q \neq j$, $\mathcal{F}_{q} \cap \mathcal{F}_{j}=\varnothing$, where $\varnothing$ denotes an empty set. The above properties imply that no two users share the same set of frequencies. Hence, there is no multiuser interference;

$\left|\mathcal{F}_{q}\right|$ cardinality of $\mathcal{F}_{q}$, i.e. the number of subcarriers assigned to user $q$, satisfying $\left|\mathcal{F}_{q}\right|=K_{q}$ for $q \in \mathcal{Q}$;

$\gamma_{q, k}$ signal-to-noise ratio (SNR) or signal-to-interferenceplus-noise ratio (SINR) of data stream $k$ of user $q$.

Based on the above definitions, we can readily show that, in OFDMA systems, the SNR of the $k$ th subcarrier of user $q$ is given by $\gamma_{q, k}=P_{q, k} G_{q, k}$. For SC-FDMA systems, as shown in [9], when the one-tap minimum mean square error (MMSE) frequency-domain equalization is employed for mitigation of the ISI, the SINR of the $k$ th data stream of user $q$ is $\gamma_{q, k}=\left(1 / K_{q} \sum_{j \in \mathcal{F}_{q}}\left(P_{q, j} G_{q, j}+1\right)^{-1}\right)^{-1}-1$. Explicitly, the SNR of the OFDMA systems and the SINR of the SCFDMA systems can be expressed in a unified form as

$\gamma_{q, k}=\frac{1}{\sum_{j \in \mathcal{F}_{q}}\left|U_{j, k}\right|^{2}\left(P_{q, j} G_{q, j}+1\right)^{-1}}-1, k \in \mathcal{K}_{q}, q \in \mathcal{Q}$

where $U_{j, k}$ is the $(j, k)$ th element of a unitary matrix $U$, which satisfies $\sum_{j \in \mathcal{F}_{q}}\left|U_{j, k}\right|^{2}=1$. It can be shown that different structures of $U$ correspond to the different multiplexing methods used by the FDMA systems. Specifically, in the OFDMA systems that transmit on each subcarrier one data stream, the matrix $U$ is constituted by $Q$ number of identity sub-matrices, each of which is for one user. By contrast, in the SC-FDMA systems where one data stream is transmitted on several subcarriers, the matrix $U$ is then structured by $Q$ number of Fourier transform matrices, each of which is for one user [6].

\section{SubCARrier-Power Allocation Algorithm}

In order to improve the reliability of FDMA systems, the subcarrier-power allocation is best optimized to minimize the average error probability expressed as $P_{e}=$ $1 / M \sum_{q \in \mathcal{Q}} \sum_{j \in \mathcal{K}_{q}} P_{e}\left(\gamma_{q, j}\right)$, where $P_{e}\left(\gamma_{q, j}\right)$ denotes the error probability corresponding to a given SINR $\gamma_{q, j}$. However, this optimization problem is extremely hard to solve, due to the nonlinear relationship between $P_{e}\left(\gamma_{q, k}\right)$ and $\gamma_{q, k}$. It is wellknown that the error probability $P_{e}$ is usually dominated by the several data streams having the lowest SINR values [9]. Hence, instead of minimizing directly the average error probability $P_{e}$, we can alternatively choose to maximize the minimum (MaxMin) SINR of the data streams, i.e., to maximize the objective function $\gamma_{\min }=\min _{q \in \mathcal{Q}, k \in \mathcal{K}_{q}}\left\{\gamma_{q, k}\right\}$. Furthermore, based on (1), we can readily show that

$$
\begin{aligned}
\gamma_{\text {min }} & \geq \frac{1}{\sum_{j \in \mathcal{F}_{q}}\left|U_{j, k}\right|^{2}\left(\min _{i \in \mathcal{F}_{q}}\left\{P_{q, i} G_{q, i}\right\}+1\right)^{-1}}-1 \\
& =\frac{1}{\left(\min _{i \in \mathcal{F}_{q}}\left\{P_{q, i} G_{q, i}\right\}+1\right)^{-1}}-1=\min _{j \in \mathcal{F}_{q}}\left\{P_{q, j} G_{q, j}\right\}
\end{aligned}
$$

Therefore, for simplicity, the objective function to be maximized can be set as

$$
J=\max _{\left\{\mathcal{F}_{q}\right\},\left\{P_{q, j}\right\}}\left\{\min _{q \in \mathcal{Q}, j \in \mathcal{F}_{q}}\left\{P_{q, j} G_{q, j}\right\}\right\}
$$

which is directly related to the subcarriers' channel qualities and the corresponding power allocated to the subcarriers.

Additionally, it has been proved in [9] that the channelinversion assisted power-allocation is optimum for the MaxMin optimization problem. Furthermore, the optimum powerallocation is independent of the subcarrier-allocation scheme employed, implying that the power- and subcarrier-allocations can be carried out separately without performance loss. Since our optimization problem shown in (3) is a typical MaxMin optimization problem, therefore, our joint subcarrierpower optimization can be carried out by first considering the subcarrier-allocation and then the power-allocation.

\section{A. Subcarrier-Allocation}

The proposed subcarrier-allocation scheme aims to assign the subcarriers to the $Q$ users, so that $\min _{q \in \mathcal{Q}, j \in \mathcal{F}_{q}}\left\{G_{q, j}\right\}$ is maximized. Let us first illustrate the principles of subcarrierallocation. Let us consider a FDMA system, which employs $M=6$ subcarriers to support $Q=3$ users with each user transmitting $K_{q}=2$ data streams on two subcarriers. The channel qualities of the six subcarriers in the context of the three users are given by

$\left[\begin{array}{ccccccc}\text { Subcarrier : } & 0 & 1 & 2 & 3 & 4 & 5 \\ \text { User 0 : } & \underline{1.8} & \underline{1.7} & 1.3 & \mathbf{0 . 5} & \mathbf{0 . 3} & 0.4 \\ \text { User 1: } & 0.6 & \mathbf{0 . 7} & \underline{1.4} & \underline{1.3} & 0.8 & 0.9 \\ \text { User 2 : } & \mathbf{0 . 2} & 1.6 & \mathbf{0 . 6} & 1.2 & \underline{1.0} & \underline{\mathbf{0 . 1}}\end{array}\right]$


where the boldface number under a subcarrier represents the worst of the channel qualities associated with the three users, while the underlined numbers correspond to the subcarriers allocated to the users, when using the Greedy algorithm in [4].

In the context of the Greedy algorithm [4], for each of the users, the subcarriers are sorted in descending order based on its channel qualities. Specifically for the example of (4), the subcarriers are sorted in descending order as $\{0,1,2,3,5,4\}$, $\{2,3,5,4,1,0\}$ and $\{1,3,4,2,0,5\}$, for users 0,1 and 2 , respectively. Then, users one by one choose their most desired subcarriers from the available options. Correspondingly, for the example of (4), user 0 first selects his two best possible subcarriers, which are the zeroth and first subcarriers, from the six. Then, user 1 chooses his two best from the remainder four, which are the second and third subcarriers. Finally, user 2 has to choose the fourth subcarrier as well as the fifth subcarrier of the most unreliable, because there are no other options. Consequently, we obtain the subcarrier sets $\mathcal{F}_{0}=\{0,1\}, \mathcal{F}_{1}=\{2,3\}$ and $\mathcal{F}_{2}=\{4,5\}$, which result in that $\min _{q \in \mathcal{Q}, j \in \mathcal{F}_{q}}\left\{G_{q, j}\right\}=0.1$.

As shown in (4), one of the disadvantages of the Greedy algorithm based subcarrier-allocation is that users at the later stages are left with limited optional subcarriers to choose. The subcarrier having the worst channel quality may have to be assigned to a user. As the above example shows, at the final stage, the fifth subcarrier has to be assigned to user 2, even its corresponding channel quality of 0.1 is the worst of all. In this case, the final attainable error performance will be dominated by this worst subcarrier channel.

The WUF Greedy algorithm proposed in [5] improves the Greedy algorithm by letting the user with the worst average channel quality select its desired subcarriers first. For the above example, according to (4), the average channel qualities for users 0,1 and 2 are 1.0, 0.95 and 0.78, respectively. Hence, user 2 has the lowest average channel quality and chooses its subcarriers first, followed by user 1 and, finally, user 0 . Consequently, the subcarriers allocated to the three users are shown by the underlined numbers of the matrix

$\left[\begin{array}{ccccccc}\text { Subcarrier : } & 0 & 1 & 2 & 3 & 4 & 5 \\ \text { User 0 : } & \underline{1.8} & 1.7 & 1.3 & \mathbf{0 . 5} & \underline{\mathbf{0 . 3}} & 0.4 \\ \text { User 1: } & 0.6 & \mathbf{0 . 7} & \underline{1.4} & 1.3 & 0.8 & \underline{0.9} \\ \text { User 2: } & \mathbf{0 . 2} & \underline{1.6} & \mathbf{0 . 6} & \underline{1.2} & 1.0 & \mathbf{0 . 1}\end{array}\right]$

Therefore, we have $\mathcal{F}_{0}=\{0,4\}, \mathcal{F}_{1}=\{2,5\}, \mathcal{F}_{2}=\{1,3\}$ and $\min _{q \in \mathcal{Q}, j \in \mathcal{F}_{q}}\left\{G_{q, j}\right\}=0.3$, which is higher than 0.1 of the minimum channel quality attained by using the Greedy algorithm.

According to the above example, we can find that the WUF Greedy algorithm cannot efficiently solve the problem of assigning users the worst subcarriers. First, the user having the highest average channel quality may also be the user accommodating the subcarrier with the worst channel quality. In this case, this user may have to accept the subcarrier with the worst channel quality. Second, when a FDMA system has a high number of subcarriers experiencing highly frequencyselective fading, all the users will have a similar average channel quality, which makes the user ordering based on the average channel qualities ineffective.
In order to avoid the above problem conflicted by the Greedy and WUF Greedy algorithms, in our proposed subcarrier-allocation scheme, the subcarriers are allocated from the worst to the best, as described follows. First, considering all the users, the subcarriers are ordered from the worst to the best according to their channel qualities of all users. In detail, for the above example, the matrix after ordering is given by

$\left[\begin{array}{ccccccc}\text { Subcarrier : } & 5 & 0 & 4 & 3 & 2 & 1 \\ \text { User 0 : } & 0.4 & \underline{1.8} & \mathbf{0 . 3} & \mathbf{0 . 5} & \underline{1.3} & 1.7 \\ \text { User 1: } & \underline{0.9} & 0.6 & 0.8 & \underline{1.3} & 1.4 & \mathbf{0 . 7} \\ \text { User 2 : } & \mathbf{0 . 1} & \mathbf{0 . 2} & \underline{1.0} & 1.2 & \mathbf{0 . 6} & \underline{1.6}\end{array}\right]$

where the first column corresponds to the worst channel quality of 0.1 , the second column corresponds to the second worst channel quality of 0.2 , and so on. Based on (6), then, the subcarrier-allocation starts with the subcarrier accommodating the worst channel quality. In other words, subcarriers are allocated from left to right in (6). As shown in (6), since for the fifth subcarrier user 1 has the best channel quality, hence the fifth subcarrier is assigned to user 1 , which consequently avoids assigning user 2 this subcarrier. Then, for the zeroth subcarrier, user 0 has the best channel quality and hence is assigned this subcarrier. The above process is continued until all the users are assigned their required subcarriers. Finally, the sets of subcarriers allocated to users 0,1 , and 2 are $\mathcal{F}_{0}=\{0,2\}, \mathcal{F}_{1}=\{5,3\}$ and $\mathcal{F}_{2}=\{1,4\}$, respectively. Furthermore, the allocation yields $\min _{q \in \mathcal{Q}, j \in \mathcal{F}_{q}}\left\{G_{q, j}\right\}=0.9$, which is significantly higher than 0.1 and 0.3 of the Greedy and WUF Greedy algorithms.

It can be shown that the above-described subcarrierallocation scheme can avoid assigning a subcarrier with the worst channel quality to a user, provided that there are at least two users with different channel qualities contending the subcarrier. Hence, we refer to our subcarrier-allocation algorithm as the WSA algorithm. However, when at the end there is only one user left, it has to choose its subcarriers from the remaining subcarriers. From the above analysis, we can realize that the worst case of using the WSA algorithm occurs, when the remaining $\max \left\{K_{q}\right\}$ subcarriers allocated to the last user coincidently have the lowest channel qualities after excluding those previously avoided. However, in practice, the probability of this event should be very small even for a $M$ of moderate value. Furthermore, even in this worst case, the WSA algorithm is capable of avoiding assigning users the $\left(M-\max \left\{K_{q}\right\}\right)$ number of relatively less reliable subcarriers. This is because, except for the $\max \left\{K_{q}\right\}$ subcarriers allocated to the last user, for any of the previously allocated $\left(M-\max \left\{K_{q}\right\}\right)$ subcarriers, the WSA algorithm has optional subcarriers to choose for allocation.

In summary, the WSA subcarrier-allocation scheme can be described by the following steps:

1) Subcarrier ordering: For each subcarrier the worst channel quality of the $Q$ users is first identified, which is expressed as

$$
G_{j}^{\min }=\min _{q \in \mathcal{Q}}\left\{G_{q, j}\right\}
$$

for $j \in \mathcal{M}$. Then, the $M$ number of worst channel qualities associated with the $M$ number of subcarriers 
are arranged in ascending order, i.e., from the worst to the best, as

$$
G_{i_{0}}^{\min } \leq G_{i_{1}}^{\min } \leq \ldots \leq G_{i_{M-1}}^{\min }
$$

where $\left\{i_{0}, i_{1}, \cdots, i_{M-1}\right\}$ denotes a permutation of the subcarrier indexes in $\mathcal{M}$, which are determined by (7).

2) Subcarrier-allocation:

$\square$ (Initialization) Set $\mathcal{F}_{q}=\varnothing$ for all $q \in \mathcal{Q}$ and set $\tilde{\mathcal{Q}}=\mathcal{Q}$.

$\square$ (Allocation) For $m=0,1, \ldots, M-1$,

a) find the best user in terms of the $i_{m}$ th subcarrier: $q^{*}=\arg \max _{q \in \tilde{\mathcal{Q}}}\left\{G_{q, i_{m}}\right\}$

b) allocate the subcarrier to user $q^{*}$ : $\mathcal{F}_{q^{*}} \leftarrow \mathcal{F}_{q^{*}} \bigcup\left\{i_{m}\right\}$.

c) if $\left|\mathcal{F}_{q^{*}}\right|=K_{q^{*}}$, user $q^{*}$ is removed from $\tilde{\mathcal{Q}}$ : $\tilde{\mathcal{Q}} \leftarrow \tilde{\mathcal{Q}}-\left\{q^{*}\right\}$.

Finally, after the subcarrier-allocation is completed, the optimization problem of (3) is reduced to a pure powerallocation problem, which is analyzed in the next subsection.

\section{B. Power-Allocation}

With the channel-inversion assisted power-allocation, the power allocated to a subcarrier of a user is inversely proportional to the subcarrier channel's quality. The power-allocation algorithm can be described as follows for the uplink and downlink, respectively.

1) Uplink Power-Allocation: In the context of the uplink power-allocation, the transmission power assigned to a subcarrier of a user can be allocated under the constraint of the total transmission power of the user. Correspondingly, the optimization problem can be formed as

$$
J_{q}=\max _{\left\{P_{q, j}\right\}}\left\{\min _{j \in \mathcal{F}_{q}}\left\{P_{q, j} G_{q, j}\right\}\right\} \text { s.t. } \sum_{j \in \mathcal{F}_{q}} P_{q, j} \leq P_{q} .
$$

for any $q \in \mathcal{Q}$. In (9), $G_{q, j}, j \in \mathcal{F}_{q}$, are the channel qualities of the subcarriers allocated to the $q$ th user.

Upon solving the above optimization problem, we can show that the power allocated to subcarrier $j$ of user $q$ is given by

$$
P_{q, j}=\left\{\begin{array}{l}
P_{q}\left(\sum_{i \in \mathcal{F}_{q}} G_{q, i}^{-1}\right)^{-1} G_{q, j}^{-1}, j \in \mathcal{F}_{q} \\
0, j \notin \mathcal{F}_{q}
\end{array}\right.
$$

where $q \in \mathcal{Q}$.

Upon substituting (10) into (1), we can obtain the attainable SINR for detection of the $k$ th data stream of the $q$ th uplink user, which is

$$
\gamma_{q, k}=\gamma_{q}=P_{q}\left(\sum_{i \in \mathcal{F}_{q}} G_{q, i}^{-1}\right)^{-1}, q \in \mathcal{Q}
$$

Equation (11) shows that the SINR of user $q$ is independent of the index $k$ as well as the multiplexing method determined by the matrix $U$ as seen in (1). Therefore, all the data streams of a given uplink user achieve the same SINR. No matter whether the SC-FDMA or OFDMA communication scheme is employed, a given uplink user is capable of attaining the same error performance.
2) Downlink Power-Allocation: The downlink powerallocation is constrained by the total transmission power $P$. In this case, the optimization problem can be formed as

$$
\begin{aligned}
J=\max _{\left\{P_{q, j}\right\}}\left\{\min _{q \in \mathcal{Q}, j \in \mathcal{F}_{q}}\left\{P_{q, j} G_{q, j}\right\}\right\} \\
\text { s.t. } \sum_{q \in\{\mathcal{Q}\}, j \in \mathcal{F}_{q}} P_{q, j} \leq P .
\end{aligned}
$$

where $\left\{G_{q, j}\right\}$ are the channel qualities of the subcarriers allocated to the $Q$ number of downlink users. Upon solving this optimization problem, we obtain the transmission power allocated to subcarrier $j$ of user $q$, which is

$$
P_{q, j}=P\left(\sum_{n \in \mathcal{Q}} \sum_{i \in \mathcal{F}_{n}} G_{n, i}^{-1}\right)^{-1} G_{q, j}^{-1}, j \in \mathcal{F}_{q}, q \in \mathcal{Q} .
$$

When substituting (13) into (1), we obtain the downlink SINR of the $k$ th data stream of the $q$ th downlink user, which can be expressed as

$$
\gamma_{q, k}=\gamma=P\left(\sum_{n \in \mathcal{Q}} \sum_{i \in \mathcal{F}_{q}} G_{n, i}^{-1}\right)^{-1} .
$$

Explicitly, it is independent of the indexes $k$ and $q$, implying that all the data streams of all the downlink users achieve the same SINR and, hence, the same error performance. Furthermore, as the uplink, the SINR of (14) is independent of the multiplexing scheme $U$, implying that both the OFDMA and SC-FDMA systems are capable of attaining the same error performance.

\section{Complexity Analysis}

In this section, we analyze and compare the complexity of the proposed and some existing subcarrier-power allocation algorithms. Note first that, when the channel-inversion assisted power-allocation is employed, as shown in (10) for the uplink or (13) for the downlink, the complexity of power-allocation with respect to any subcarrier-allocation schemes is the same, which is $\mathcal{O}\left(\sum_{q \in \mathcal{Q}} K_{q}\right) \triangleq \mathcal{O}(M)$ for both the uplink and downlink power-allocation. Therefore, when comparing the complexity of different subcarrier-power allocation schemes, we only need to consider the complexity of the subcarrierallocation schemes invoked.

It is well-known that the number of comparisons to find the maximum of $M$ real numbers using binary-search is $(M-$ 1 ), while the average number of comparisons to sort $M$ real numbers using the quick-sort algorithm [12] is $2 M \ln M$. Our proposed WAS algorithm requires to find both the minimum and maximum of the $Q$ number of channel qualities for each of the $M$ number of subcarriers. Furthermore, it also requires to order the $M$ number of worst channel qualities. Hence, the total number of operations is about $(2 M(Q-1)+2 M \ln M)$, yielding a complexity of $\mathcal{O}(M Q)$ when assuming $Q \geq \ln M$.

The exact number of operations required by the Hungarian algorithm [3] is very hard to analyze, due to the randomness of the cost matrices invoked and the heuristic nature of the algorithm [6]. In [11], the maximum number of operations required by the Hungarian algorithm has been analyzed, which is found to be $\left(11 M^{3}+12 M^{2}+31 M\right) / 6$. Hence, the complexity of the Hungarian algorithm is $\mathcal{O}\left(M^{3}\right)$. As shown in Section III-A, 
TABLE I

COMPLEXITY COMPARISON OF VARIOUS SUBCARRIER ALLOCATION ALGORITHMS.

\begin{tabular}{c|c|c}
\hline \hline Algorithm & Number of operations & Complexity \\
\hline Hungarian [3, 11] & $\left(11 M^{3}+12 M^{2}+31 K\right) / 6$ & $\mathcal{O}\left(M^{3}\right)$ \\
\hline Greedy [4] & $M(M-1) / 2$ & $\mathcal{O}\left(M^{2}\right)$ \\
\hline WUF Greedy [5] & $M(M-1) / 2+2 Q \ln Q$ & $\mathcal{O}\left(M^{2}\right)$ \\
\hline Wong, et.al [7] & $M(M-1) / 2+a M^{2} / 2$ & $\mathcal{O}\left(M^{2}\right)$ \\
\hline Maximal Greedy [6] & $Q M(M-1) / 2$ & $\mathcal{O}\left(Q M^{2}\right)$ \\
\hline WSA (Proposed) & $2 M \ln M+2 Q M$ & $\mathcal{O}(Q M)$ \\
\hline \hline
\end{tabular}

the Greedy algorithm [4] only requires to find the maximum of the available subcarriers, one-by-one, for all the users. The corresponding number of operations required can be found to be $(M-1) \sum_{q \in \mathcal{Q}} K_{q} / 2$, which is $(M-1) M / 2$, when all the $M$ number of subcarriers are allocated, i.e., when $M=\sum_{q \in \mathcal{Q}} K_{q}$. Therefore, the complexity of the Greedy algorithm is $\mathcal{O}\left(M^{2}\right)$. In comparison with the Greedy algorithm [4], as shown in Section III-A, the WUF Greedy algorithm [5] requires $(2 Q \ln Q)$ extra operations for sorting the $Q$ users, in addition to the operations required by the Greedy algorithm. Hence, the WUF Greedy algorithm requires a total $(M(M-1) / 2+2 Q \ln Q)$ number of operations, making its complexity $\mathcal{O}\left(M^{2}\right)$.

The method proposed by Wong, et.al, in [7] needs to swap iteratively the subcarriers between two users chosen arbitrary from $Q$ users. It has been shown [8] that the number of operations per iteration is $C_{2}^{Q} K_{q}^{2} \approx M^{2} / 2$. Thus, when using $a$ iterations, the total number of operations is $\left(M(M-1) / 2+a M^{2} / 2\right)$ and the complexity is hence $\mathcal{O}\left(M^{2}\right)$. Note that, the results in [7] demonstrate that this method is near-optimum provided that $a \geq 25$ iterations are used, and can perform reasonably well, when using about $a=10$ iterations. As shown in [4], the number of operations required by the Maximal Greedy algorithm [6] is $Q M(M-1) / 2$, which is $Q$ times of that of the Greedy algorithm. Hence, the Maximal Greedy algorithm has a complexity of $\mathcal{O}\left(Q M^{2}\right)$.

Finally, the complexity of the above-addressed subcarrier allocation schemes is summarized in Table I. Furthermore, the number of operations required by the various subcarrierallocation schemes is compared in Fig. 1, when the FDMA systems employ different number of subcarriers, each user uses $K_{q}=8$ subcarriers and the total number of users is $Q=M / K_{q}$. As shown in Fig. 1, the proposed WSA algorithm requires slightly more operations than the Greedy and WUF Greedy algorithms when $M \leq 32$ subcarriers are employed by the FDMA system, while has a slightly lower number of operations than the two Greedy algorithms when $M>32$ subcarriers are employed. Furthermore, from the results of Fig. 1, we are implied that the WSA algorithm has a similar complexity as the Greedy algorithm or the WUF Greedy algorithm. These three subcarrier-allocation algorithms have the lowest complexity among those considered in this contribution.

\section{Performance Results}

In this section we compare the average BER performance of the proposed algorithm with that of several existing algorithms as listed in Table I, when an OFDMA system using $M=32$

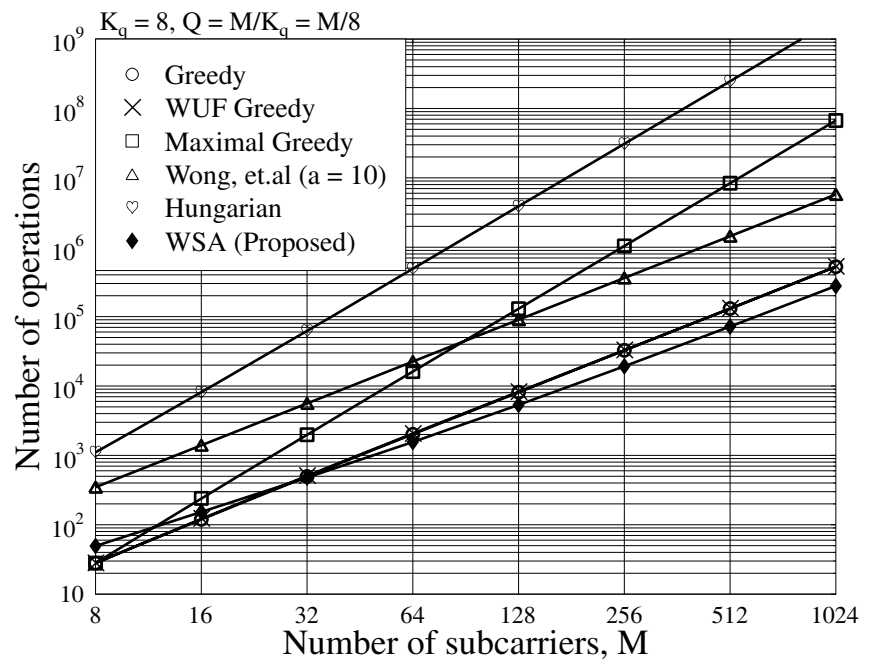

Fig. 1. Number of operations versus the total number of subcarriers for the FDMA systems employing different subcarrier-allocation schemes.

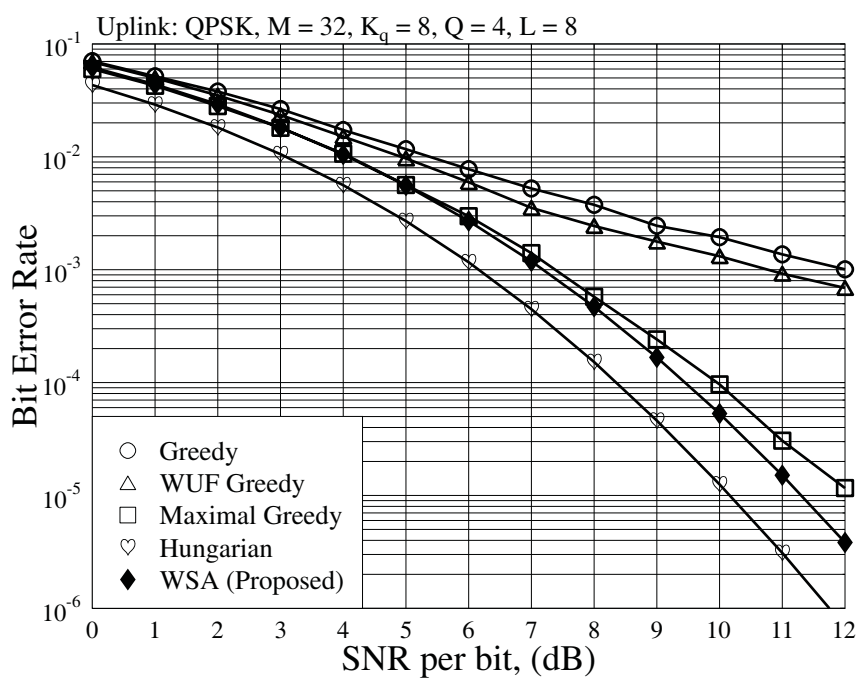

Fig. 2. Uplink BER versus SNR per bit performance of OFDMA systems experiencing frequency-selective Rayleigh fading with $L=8$ time-domain resolvable paths.

subcarriers to support $Q=4$ users is considered. We assume that each user is assigned $K_{q}=M / Q=8$ subcarriers for transmitting eight data streams using Quadrature Phase-Shift Keying (QPSK) baseband modulation. The communications channels are assumed the frequency-selective Rayleigh fading channels that have $L=8$ time-domain resolvable paths.

Note that, although our simulations were based on the OFDMA scheme, the results as shown are actually suitable for the SC-FDMA scheme using $M=32$ subcarriers to support $Q=4$ users with each user transmitting $K_{q}=8$ data streams. This is because, when employing the MaxMin optimization, the error performance is independent of the multiplexing scheme for all the subcarrier-power allocation algorithms considered.

Figure 2 shows the BER performance of the OFDMA uplink employing various subcarrier-allocation algorithms, when individual power constraint is applied and the transmission power is allocated based on (10) for all the algorithms. 


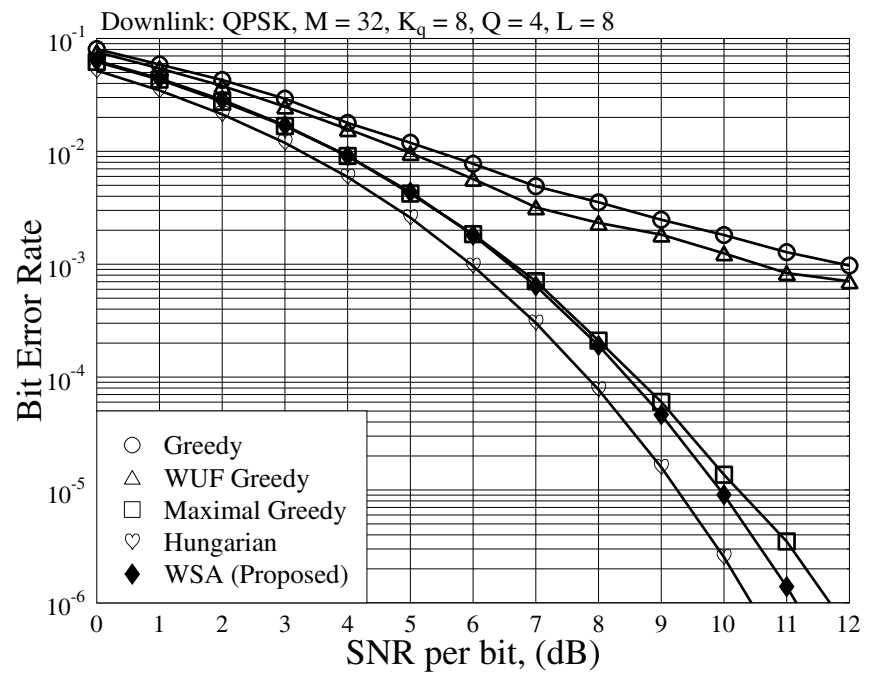

Fig. 3. Downlink BER versus SNR per bit performance of OFDMA systems experiencing frequency-selective Rayleigh fading with $L=8$ time-domain resolvable paths.

By contrast, Fig. 3 illustrates the BER performance of the OFDMA downlink employing various subcarrier-allocation algorithms, when the total transmission power is constrained and the power is allocated to a subcarrier of a user according to $(13)$.

From the results, it can be observed that our proposed algorithm outperforms the Greedy, WUF Greedy and the Maximal Greedy algorithm. The BER performance curve of the proposed algorithm is close to and also parallel to that of the Hungarian algorithm, which is argued to be optimum [7]. However, as shown in Table I, the Hungarian algorithm has the highest complexity among the algorithms considered. Furthermore, when comparing the results in Fig. 2 with that in Fig. 3, we can see that, for a given algorithm, the downlink is capable of achieving better BER performance than the uplink. This is because the power-allocation for the downlink considers jointly all the users, while that for the uplink considers user-by-user separately.

\section{CONCLUSIONS}

In this contribution we have proposed and investigated a so-called WSA-assisted subcarrier-allocation scheme and a channel-inversion assisted power-allocation scheme, in order to improve the reliability of various types of FDMA systems. The achievable error performance of the FDMA systems employing the proposed algorithm is the same, regardless of the multiplexing method employed. The proposed algorithm has low complexity and, in terms of the achievable error performance, outperforms the existing subcarrier-power allocation algorithms that have a similar complexity as the proposed algorithm.

\section{REFERENCES}

[1] K. B. Letaief and Y. J. Zhang, "Dynamic multiuser resource allocation and adaptation for wireless systems," IEEE Wireless Commun. Mag., vol. 13, no. 4, pp. 38-47, Aug. 2006.

[2] G. Song and Y. Li, "Cross-layer optimization for OFDM wireless networks-part I: theoretical framework," IEEE Trans. Wireless Commun., vol. 4, no. 2, pp. 614-624, Mar. 2005.

[3] H. W. Kuhn, "The Hungarian method for the assignment problem," Naval Research Logistics Quarterly, vol. 2, pp. 83-97, 1955.

[4] Y. Kim and J. Kim, "A 2-D subcarrier allocation scheme for capacity enhancement in a clustered OFDM system," IEICE Trans. Commun., vol. E90-B, no. 7, pp. 1880-1883, July 2007.

[5] N. Y. Ermolova and B. Makarevitch, "Performance of practical subcarrier allocation schemes for OFDMA," in Proc. IEEE PIMRC 2007, Sept. 2007, pp. 1-4.

[6] O. Nwamadi, X. Zhu, and A. Nandi, "Dynamic subcarrier allocation for single carrier - FDMA systems," in Proc. EUSIPCO 2008, Aug. 2008.

[7] C. Y. Wong, C. Y. Tsui, R. S. Cheng, and K. B. Letaief, "A realtime sub-carrier allocation scheme for multiple access downlink OFDM transmission," in Proc. IEEE VTC 1999-Fall, vol. 2, Sept. 1999, pp. 1124-1128.

[8] Y.-F. Chen and J.-W. Chen, "A fast subcarrier, bit, and power allocation algorithm for multiuser OFDM-based systems," IEEE Trans. Veh. Technol., vol. 57, no. 2, pp. 873-881, Mar. 2008.

[9] D. P. Palomar, J. M. Cioffi, and M. A. Lagunas, "Joint Tx-Rx beamforming design for multicarrier MIMO channels: a unified framework for convex optimization," IEEE Trans. Signal Process., vol. 51, no. 9, pp. 2381-2401, Sept. 2003.

[10] D. E. Knuth, The Art of Computer Programming Volume 3: Sorting and Searching. Reading, MA: Addison-Wesley, 1998.

[11] J. Munkres, "Algorithms for the assignment and transportation problems," J. Society for Industrial and Applied Mathematics, vol. 5, pp. 32-38, 1957.

[12] W.H. Press, et.al, Numerical Recipes in C: The Art of Scientific Computing (2nd Ed). Cambridge University Press, 1992. 